\title{
Relationship between stroke etiology and collateral status in anterior circulation large vessel occlusion
}

\author{
Eva Hassler ${ }^{1} \cdot$ Markus KneihsI $^{2} \cdot$ Hannes Deutschmann ${ }^{1} \cdot$ Nicole Hinteregger $^{1} \cdot$ Marton Magyar $^{1}$. \\ Ulrike Wießpeiner ${ }^{1} \cdot$ Melanie Haidegger $^{2} \cdot$ Simon Fandler-Höfler ${ }^{2} \cdot$ Sebastian Eppinger $^{2} \cdot$ Kurt Niederkorn $^{2}$. \\ Christian Enzinger ${ }^{1,2} \cdot$ Franz Fazekas $^{2} \cdot$ Thomas Gattringer $^{1,2}[$
}

Received: 5 March 2020 / Revised: 17 June 2020 / Accepted: 18 June 2020 / Published online: 25 June 2020

(c) The Author(s) 2020

\begin{abstract}
Background and purpose Clinical outcome after mechanical thrombectomy (MT) for large vessel occlusion (LVO) stroke is influenced by the intracerebral collateral status. We tested the hypothesis that patients with preexisting ipsilateral extracranial carotid artery stenosis (CAS) would have a better collateral status compared to non-CAS patients. Additionally, we evaluated MT-related adverse events and outcome for both groups.

Methods Over a 7-year period, we identified all consecutive anterior circulation MT patients (excluding extracranial carotid artery occlusion and dissection). Patients were grouped into those with CAS $\geq 50 \%$ according to the NASCET criteria and those without significant carotid stenosis (non-CAS). Collateral status was rated on pre-treatment CT- or MR-angiography according to the Tan Score. Furthermore, we assessed postinterventional infarct size, adverse events and functional outcome at 90 days.

Results We studied 281 LVO stroke patients, comprising 46 (16.4\%) with underlying CAS $\geq 50 \%$. Compared to non-CAS stroke patients $(n=235)$, patients with CAS-related stroke more often had favorable collaterals $(76.1 \%$ vs. $46.0 \%)$. Recanalization rates were comparable between both groups. LVO stroke patients with underlying CAS more frequently had adverse events after MT (19.6\% vs. 6.4\%). Preexisting CAS was an independent predictor for favorable collateral status in multivariable models (Odds ratio: 3.3, $p=0.002$ ), but post-interventional infarct size and functional 90-day outcome were not different between CAS and non-CAS patients.

Conclusions Preexisting CAS $\geq 50 \%$ was associated with better collateral status in LVO stroke patients. However, functional 90-day outcome was independent from CAS, which could be related to a higher rate of adverse events.
\end{abstract}

Keywords Stroke $\cdot$ Collateral circulation $\cdot$ Thrombectomy $\cdot$ Carotid artery diseases $\cdot$ Outcome

\section{Introduction}

Eva Hassler, Markus Kneihsl contributed equally to the manuscript.

Thomas Gattringer

thomas.gattringer@medunigraz.at

1 Division of Neuroradiology, Vascular and Interventional Radiology, Department of Radiology, Medical University of Graz, Graz, Austria

2 Department of Neurology, Medical University of Graz, Auenbruggerplatz 22, 8036 Graz, Austria
Mechanical thrombectomy (MT) is the recommended treatment for acute ischemic stroke due to large vessel occlusion (LVO) of the anterior cerebral circulation [1]. With increasing experience and technical advances, successful recanalization can nowadays be achieved in up to $90 \%$ of all thrombectomy cases. However, successful recanalization does not always entail a favorable outcome after endovascular stroke treatment. In this context, the extent of leptomeningeal collateral perfusion has been identified as a major determinant of patients' clinical prognosis [2]. Unfavorable collateral status on preinterventional angiography has been related to larger final infarct volumes and consequently a worse clinical outcome after MT [2,3]. 
Previously, it has been assumed that chronically developing extracranial carotid artery stenosis (CAS) could enhance cerebral collateral flow, which might be related to a more favorable prognosis in acute stroke patients [4]. This raised the question, whether an underlying CAS would also lead to better leptomeningeal collaterals in patients with acute anterior circulation LVO stroke compared to those with more abrupt vessel occlusion due to proximal embolism (e.g. cardiogenic embolism from atrial fibrillation).

While small previous investigations showed inconsistent results on the predictive value of stroke etiology on collateral status in LVO patients, [5-7] a very recent subanalysis of the MR-CLEAN Registry reported higher collateral recruitment in acute stroke patients with an underlying atherosclerotic carotid artery stenosis [8]. However, for yet unknown reasons, this might not translate into a higher chance for CAS patients to achieve functional independency after stroke [8].

We aimed at investigating preinterventional leptomeningeal collateral status in acute LVO patients according to the underlying putative stroke mechanism (atherosclerotic CAS $\geq 50 \%$ versus patients without significant ipsilateral CAS) and how this affects postinterventional adverse events and clinical outcome.

\section{Materials and methods}

\section{Patient selection and data collection}

For the present study, we identified all consecutive ischemic stroke patients aged $\geq 18$ years, who were treated by MT for acute anterior circulation LVO (i.e. occlusion of the intracranial internal carotid artery or middle cerebral artery in the M1 or M2 segment) between 2010 and 2017 at our primary and tertiary care university hospital.

Clinical data including demographics, cerebrovascular risk factors, stroke etiology, characteristics of the endovascular procedure and outcome were retrieved from our prospectively collected electronical thrombectomy database [9].

Patients were divided into those with an underlying atherosclerotic extracranial ipsilateral carotid artery stenosis $\geq 50 \%$ (CAS) and those without an indication of preexisting significant carotid steno-occlusive disease (non-CAS). The cut-off was chosen because it (1) corresponds to recent guideline recommendations for diagnosing symptomatic carotid artery stenosis [10] and (2) was also used in prior studies on this topic [8]. Presence and degree of stenosis was determined on preinterventional computed tomography (CT) or magnetic resonance imaging (MRI) based contrast enhanced (CE) angiography and confirmed by digital subtraction angiography during the thrombectomy procedure using the North American Symptomatic Carotid Endarterectomy Trial (NASCET) criteria [11]. Patients with extracranial carotid artery occlusion were excluded from the study, as it was not possible to determine whether they had a preexisting stenosis (Fig. 1).

Mechanical thrombectomy was conducted by interventional radiologists using stent retrievers and/or aspiration systems. If ipsilateral CAS was present, acute stenting procedures were performed at the discretion of the treating physician depending on morphology and grade of carotid stenosis (e.g. high-risk stenosis due to ulcerated plaque, visible residual thrombi or filiform stenosis).

\section{Imaging work-up and analyzes}

All included patients underwent preinterventional brain imaging including intra- and extracranial CT or MRI based CE angiography (CT angiography: $\approx 90 \%$ ).

Postinterventional control brain imaging (predominantly MRI) was routinely performed $24 \mathrm{~h}$ after thrombectomy or at any time in case of clinical deterioration.

All images were retrospectively analyzed by two experienced neuroradiologists (E.H., M.M.), who were blinded to clinical and outcome data.

Leptomeningeal collateral status on preinterventional CT- or MR-angiography was categorized according to the collateral score by Tan et al. into scores 0 : absent collateral supply of the affected MCA territory, 1: collateral supply filling $\leq 50 \%, 2$ : collateral supply filling $>50 \%$ but $<100 \%$, and $3: 100 \%$ collateral supply of the occluded MCA territory [12].

Leptomeningeal collaterals were furthermore dichotomized into unfavorable collaterals (collateral score: $0-1$ ) and favorable collaterals (collateral score: 2-3) (Fig. 2) [5].

\section{Postinterventional adverse events and outcome}

Postinterventional brain scans were reviewed to identify intracranial hemorrhage (ICH). ICH after MT was defined according to the Heidelberg Bleeding Classification and deemed symptomatic if a deterioration of patient's clinical symptoms was observed (defined as a National Institutes of Health Stroke Scale [NIHSS] score increase of $>2$ points in one category or $>4$ points in total) $[13,14]$. Treatmentrelated arterial re-occlusion or dissection was diagnosed by digital subtraction angiography in synopsis with postinterventional CT/MR-angiography and color-coded duplex sonography of the extra- and intracranial vessels.

Functional neurological outcome according to the modified Rankin Scale (mRS) was assessed by a neurologist with special expertise in stroke in a personal visit at the stroke outpatient department or if not possible in a telephone interview at 90 days poststroke. 


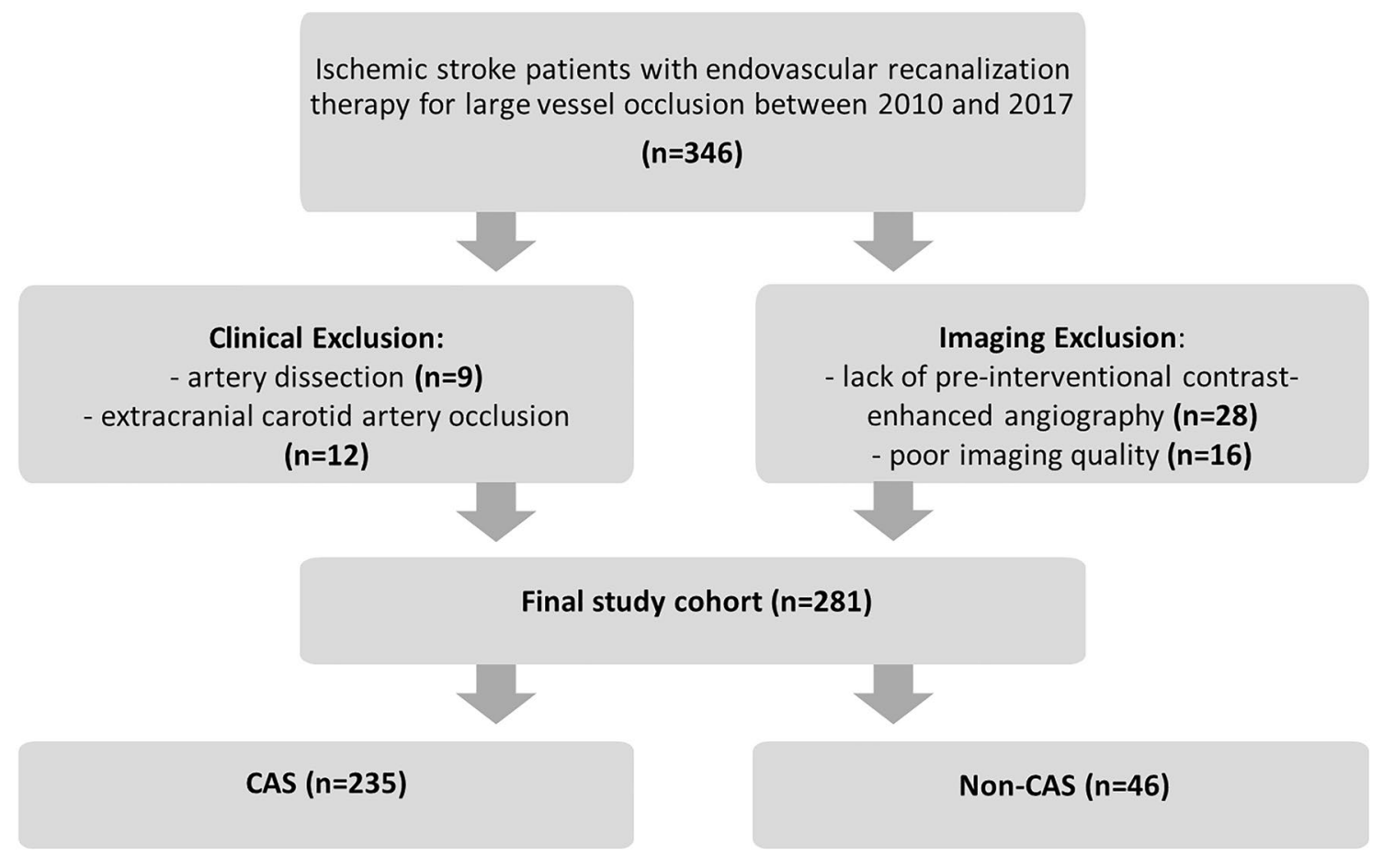

Fig. 1 Flow diagram of included study participants

\section{Statistics}

Statistical analyses were performed using IBM SPSS Statistics, version 23. The association between stroke etiology (CAS versus non-CAS) and preinterventional leptomeningeal collateral status was investigated.

Chi square test or Fisher's exact test was used for the comparison of dichotomous variables. Parametric continuous variables were compared using the Student's $t$ test. For nonparametric data, the Mann-Whitney $U$ Test was utilized.

In addition, we calculated a multivariable binary logistic regression model with favorable collaterals as the target variable. Besides CAS, it also contained age, sex and other previously identified predictors of collateral status [hypertension, M2-occlusion, baseline NIHSS and baseline Alberta Stroke Program Early CT Score (ASPECTS)] [5-7].

A $p$ value less than 0.05 was considered statistically significant.

The study was approved by the ethics committee of the Medical University of Graz. Anonymized datasets generated during this study are available from the corresponding author upon reasonable request.

\section{Results}

Over the study period, 346 patients had undergone MT for anterior circulation LVO. Of those, 65 patients were excluded due to missing $\mathrm{CE}$ angiography data or insufficient imaging quality $(n=44)$, ipsilateral carotid artery dissection $(n=9)$ or extracranial occlusion $(n=12)$ (Fig. 1). None of the studied patients had a significant intracranial artery stenosis.

Of the finally analyzed 281 patients (mean age 68.5 years, $48.8 \%$ female, Table 1), 46 patients had CAS-associated LVO stroke (16.4\%).

The remaining 235 patients $(83.6 \%)$ showed a nonCAS stroke that was mainly attributed to atrial fibrillation $(58.8 \%)$. Of those, no patients with ulcerated high-risk non-stenosing carotid artery plaques were observed. Compared to a non-CAS etiology, CAS-related strokes were less prevalent in female patients ( $32.6 \%$ vs. $51.9 \%, p=0.012$ ), more often pretreated with antiplatelet agents $(39.1 \%$ vs. $23.4 \%, p=0.023)$ and had a higher rate of favorable collaterals on pretreatment angiography $(76.1 \%$ vs. $46.0 \%$, $p<0.001)$. Patients with underlying CAS more often 


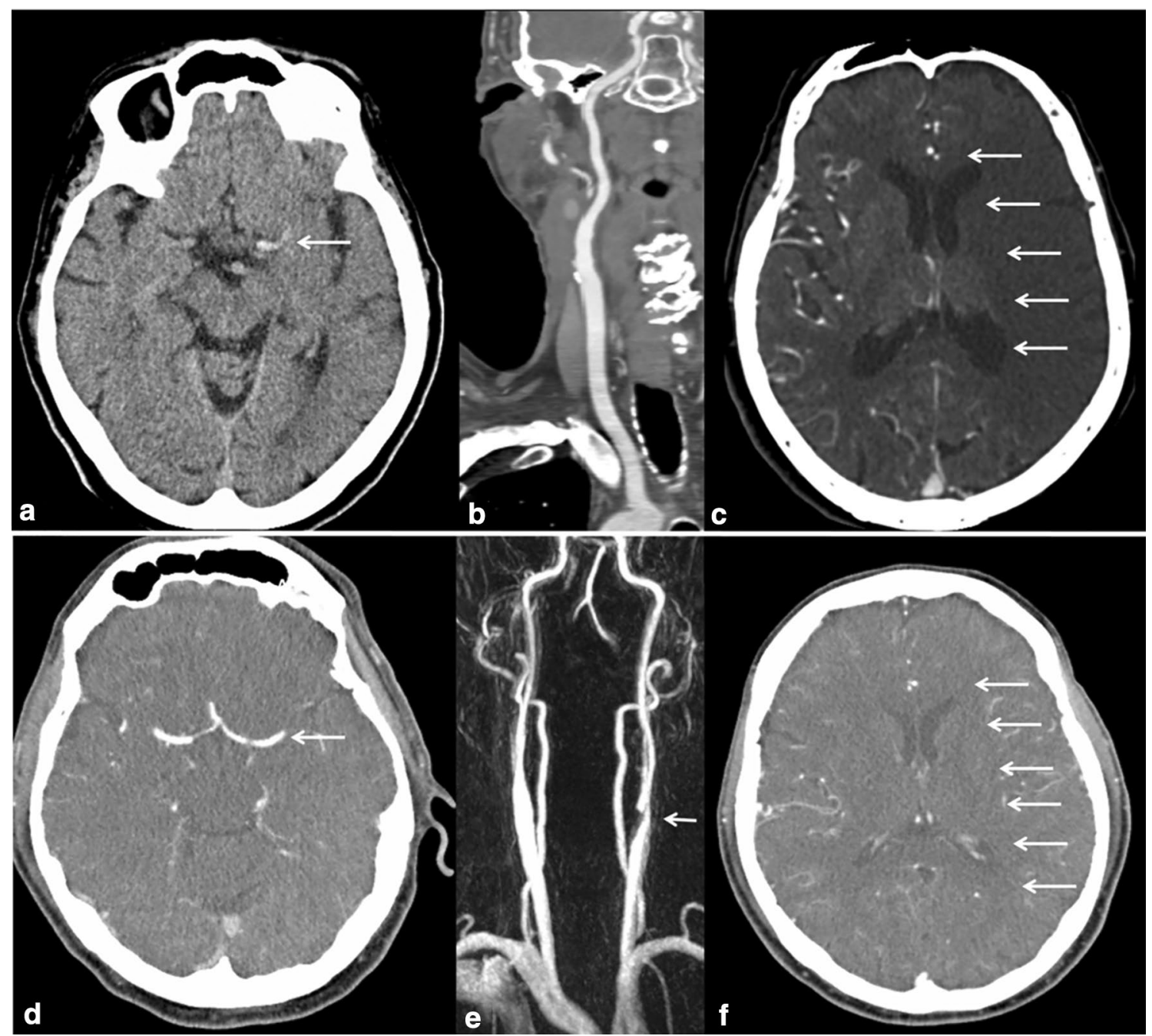

Fig. 2 Exemplary cases of acute LVO stroke patients with good and poor collaterals. a Shows left sided hyperdense media sign (arrow) on preinterventional CT scan of a patient with acute occlusion of the M1 segment of the middle cerebral artery without an indication of ipsilateral carotid artery stenosis (b). Intracranial CT angiography (CTA) displays unfavorable collateral status according to a Tan collateral score of 0 before thrombectomy (c, arrows). Another patient presents with acute left sided atheroembolic M1 occlusion (d, arrow) and high-grade extracranial carotid artery stenosis on preinterventional CTA, which is demonstrated in e (arrow). $\mathbf{f}$ shows favorable collaterals according to a Tan collateral score of 3 (arrows)

less likely deceased at 90 days (mRS 6: $7.0 \%$ vs. $29.7 \%$, $p<0.001$ ) (Table 2).

Besides NIHSS at presentation $(p<0.001)$ and preinterventional ASPECTS $(p=0.033)$, favorable collateral status remained significantly associated with CAS-related stroke etiology in multivariable analysis (Odds ratio: $3.3,95 \%$ confidence interval: $1.5-7.1, p=0.002$ ).

\section{Unfavorable collateral status and stroke etiology}

138 patients had unfavorable collaterals on pre-treatment angiography $(49.1 \%)$. Of those, only 11 patients had CAS-associated stroke (8.0\%). Compared to non-CAS patients with an unfavorable collateral status $(n=127)$, pendent (mRS $0-2: 59.4 \%$ vs. $35.5 \%, p<0.001)$ and were 
Table 1 Clinical characteristics of anterior circulation LVO stroke patients dichotomized by stroke etiology

MT of the anterior circu- CAS $(n=46) \quad$ Non-CAS $(n=235) \quad p$ value
lation $(n=281)$

\section{Demographics}

Age, years (mean, SD)

Female [n. \%]

Medical history ( $n . \%)$

Hypertension

Dyslipidemia

Diabetes

Smoking

Atrial fibrillation

Previous antiplatelet therapy

Previous oral anticoagulation

Prestroke mRS (n. \%)

0

1

$\geq 2$

Clinical parameters

NIHSS at presentation (median, range)

Preinterventional ASPECTS (median, min-max)

Symptom onset - conventional angiography (minutes; mean, SD)

Symptom onset-reperfusion (minutes; mean, SD)

Duration of intervention (minutes; mean, SD)

Favorable collaterals

Postinterventional ASPECTS (median, min-max)

Infarct $\geq 2 / 3$ MCA territory ( $n$. \%)

Acute stroke therapy ( $n$. \%)

IV thrombolysis

MCA thrombectomy

Successful mechanical thrombectomy (TICI 2b-3)

Extracranial artery stenting ${ }^{a}$

Outcome and adverse events ( $n$. \%)

NIHSS at $24 \mathrm{~h}$ (median, range)

NIHSS at stroke unit discharge (median, range)

Adverse events

Symptomatic ICH

Procedure-related artery dissection

Vessel re-occlusion

Mortality at hospital discharge

90-day mRS (median, IQR)

90-day mRS 0-2

90-day mRS 3-5

90-day mRS 6

\begin{tabular}{|c|c|c|c|}
\hline $68.5 \pm 12.2$ & $67.0 \pm 11.2$ & $68.8 \pm 12.4$ & 0.342 \\
\hline $137(48.8)$ & $15(32.6)$ & $122(51.9)$ & 0.012 \\
\hline $193(68.7)$ & $32(69.6)$ & $161(68.5)$ & 0.519 \\
\hline $51(18.1)$ & 9 (19.6) & $42(17.9)$ & 0.463 \\
\hline $50(17.8)$ & $7(15.2)$ & $43(18.3)$ & 0.398 \\
\hline $31(11.0)$ & $7(15.2)$ & $24(10.2)$ & 0.225 \\
\hline $141(50.2)$ & $4(8.7)$ & $137(58.8)$ & $<0.001$ \\
\hline $73(26.0)$ & $18(39.1)$ & $55(23.4)$ & 0.023 \\
\hline $40(14.2)$ & $3(6.5)$ & $37(15.7)$ & 0.073 \\
\hline & & & 0.335 \\
\hline $238(85.0)$ & $41(89.1)$ & $198(84.3)$ & \\
\hline $14(5.0)$ & $4(8.7)$ & $10(4.3)$ & \\
\hline $28(10.0)$ & $1(2.2)$ & $27(11.4)$ & \\
\hline $15(4-32)$ & $14(7-22)$ & $15(4-32)$ & 0.068 \\
\hline $9.0(5-10)$ & $9.0(6-10)$ & $9.0(5-10)$ & 0.848 \\
\hline $199 \pm 74$ & $197 \pm 85$ & $200 \pm 72$ & 0.836 \\
\hline $257 \pm 76$ & $264 \pm 83$ & $255 \pm 75$ & 0.555 \\
\hline $58 \pm 34$ & $67 \pm 39$ & $56 \pm 33$ & 0.081 \\
\hline $143(50.9)$ & $35(76.1)$ & $108(46.0)$ & $<0.001$ \\
\hline $5.0(0-10)$ & $5.5(0-9)$ & $5.0(0-10)$ & 0.453 \\
\hline $49(17.4)$ & 9 (19.6) & $40(17.0)$ & 0.408 \\
\hline $166(59.1)$ & $25(54.3)$ & $141(60.0)$ & 0.290 \\
\hline $232(82.6)$ & $35(76.1)$ & $197(83.8)$ & 0.146 \\
\hline 252 (89.7) & $41(89.1)$ & $211(89.8)$ & 0.533 \\
\hline $11(3.9)$ & $11(23.9)$ & $0(0.0)$ & $<0.001$ \\
\hline $10(0-32)$ & $12.5(1-32)$ & $9(0-32)$ & 0.291 \\
\hline $8.5(0-32)$ & $6.5(0-32)$ & $8(0-32)$ & 0.141 \\
\hline $24(8.5)$ & 9 (19.6) & $15(6.4)$ & 0.007 \\
\hline $10(3.6)$ & $3(6.5)$ & $7(3.1)$ & 0.231 \\
\hline $3(1.1)$ & $1(2.2)$ & $2(0.9)$ & 0.416 \\
\hline $11(3.9)$ & $5(10.9)$ & $6(2.6)$ & 0.020 \\
\hline $30(10.7)$ & $3(6.5)$ & $27(11.5)$ & 0.238 \\
\hline $3(3)$ & $3(3)$ & $3(3)$ & 0.861 \\
\hline $134(47.7)$ & $21(45.7)$ & $113(48.1)$ & 0.445 \\
\hline $96(34.2)$ & $20(43.5)$ & $76(32.3)$ & 0.100 \\
\hline $51(18.1)$ & 5 (10.9) & 46 (19.6) & 0.114 \\
\hline
\end{tabular}

MT mechanical thrombectomy, CAS Carotid artery stenosis, SD Standard deviation, NIHSS National Institutes of Health Stroke Scale, ASPECTS Alberta Stroke Program Early CT Score, $M C A$ Middle cerebral artery, TICI Thrombolysis in cerebral infarction grading scale, ICH Intracranial hemorrhage, $m R S$ Modified Rankin scale, IQR Interquartile range

${ }^{a} 10$ patients underwent carotid artery stenting simultaneously with thrombectomy; one patient was treated later on (day 38 ) 
Table 2 Clinical characteristics of LVO stroke patients according to their preinterventional collateral status

\begin{tabular}{|c|c|c|c|}
\hline & $\begin{array}{l}\text { Favorable collat- } \\
\text { erals }(n=143)\end{array}$ & $\begin{array}{l}\text { Unfavorable col- } \\
\text { laterals }(n=138)\end{array}$ & $p$ value \\
\hline \multicolumn{4}{|l|}{ Demographics } \\
\hline Age, years (mean, SD) & $66.6 \pm 12.1$ & $70.6 \pm 12.1$ & 0.005 \\
\hline Female $[n . \%]$ & $69(48.3)$ & $68(49.3)$ & 0.864 \\
\hline \multicolumn{4}{|l|}{ Medical history ( $n . \%)$} \\
\hline Hypertension & $96(67.1)$ & $97(70.3)$ & 0.568 \\
\hline Dyslipidemia & $28(19.6)$ & $23(16.7)$ & 0.526 \\
\hline Diabetes & $22(15.4)$ & $28(20.3)$ & 0.282 \\
\hline Smoking & $15(10.5)$ & $16(11.6)$ & 0.768 \\
\hline Atrial fibrillation & $64(45.1)$ & $77(56.1)$ & 0.063 \\
\hline Prestroke mRS (n. \%) & & & 0.085 \\
\hline 0 & $126(88.1)$ & $113(81.9)$ & \\
\hline 1 & $10(7.0)$ & $4(2.9)$ & \\
\hline$\geq 2$ & $7(4.9)$ & $21(15.2)$ & \\
\hline \multicolumn{4}{|l|}{ Clinical parameters } \\
\hline NIHSS at presentation (median, range) & $14(4-25)$ & $16(6-32)$ & $<0.001$ \\
\hline Preinterventional ASPECTS (median, min-max) & $9.0(5-10)$ & $8.5(5-10)$ & 0.035 \\
\hline $\begin{array}{l}\text { Symptom onset-conventional angiography (minutes; } \\
\text { mean, SD) }\end{array}$ & $197 \pm 86$ & $202 \pm 58$ & 0.646 \\
\hline Symptom onset-reperfusion (minutes; mean, SD) & $254 \pm 90$ & $260 \pm 56$ & 0.560 \\
\hline Duration of intervention (minutes; mean, SD) & $57 \pm 33$ & $58 \pm 35$ & 0.785 \\
\hline Postinterventional ASPECTS (median, min-max) & $6.0(0-10)$ & $4.0(0-9)$ & $<0.001$ \\
\hline Infarct $\geq 2 / 3$ MCA territory $(n . \%)$ & $13(9.1)$ & $36(26.1)$ & $<0.001$ \\
\hline CAS $(n . \%)$ & $35(31.8)$ & $11(11.5)$ & $<0.001$ \\
\hline \multicolumn{4}{|l|}{ Acute stroke therapy $(n . \%)$} \\
\hline IV thrombolysis & $91(63.6)$ & $75(54.3)$ & 0.113 \\
\hline MCA thrombectomy & $115(80.4)$ & $117(84.8)$ & 0.335 \\
\hline Successful mechanical thrombectomy (TICI 2b-3) & $131(91.6)$ & $121(87.7)$ & 0.279 \\
\hline Extracranial artery stenting & $11(7.7)$ & $0(0.0)$ & 0.001 \\
\hline \multicolumn{4}{|l|}{ Outcome and Adverse events ( $n . \%$ ) } \\
\hline Adverse events & $10(7.0)$ & $14(10.1)$ & 0.345 \\
\hline Symptomatic ICH & $4(2.9)$ & $6(4.5)$ & 0.481 \\
\hline Procedure-related artery dissection & $2(1.4)$ & $1(0.7)$ & 0.583 \\
\hline Vessel re-occlusion & $4(2.8)$ & $7(5.1)$ & 0.326 \\
\hline Mortality at stroke unit discharge & $4(2.8)$ & $26(18.8)$ & $<0.001$ \\
\hline 90-day mRS (median, min-max) & $2(3)$ & $4(4)$ & $<0.001$ \\
\hline 90-day mRS 0-2 & $85(59.4)$ & $49(35.5)$ & $<0.001$ \\
\hline 90-day mRS 3-5 & $48(33.6)$ & $48(34.8)$ & 0.830 \\
\hline 90-day mRS 6 & $10(7.0)$ & $41(29.7)$ & $<0.001$ \\
\hline
\end{tabular}

LVO large vessel occlusion, SD standard deviation, NIHSS: National Institutes of Health Stroke Scale, ASPECTS Alberta Stroke Program Early CT Score, MCA Middle cerebral artery, CAS Carotid artery stenosis, TICI Thrombolysis in cerebral infarction grading scale, ICH Intracranial hemorrhage, $m R S$ Modified Rankin scale, IQR: Interquartile range
CAS-related stroke patients with unfavorable collaterals were associated with a particularly high rate of adverse events $(36.4 \%$ vs. $9.4 \%, p=0.025)$ and poor outcome (median NIHSS at stroke unit discharge: 21 vs. 9 , $p=0.037$; 90-day mRS $0-2: 9.1 \%$ vs. $37.8 \%, p=0.050)$.

\section{Discussion}

This study shows that the presence of preexisting ipsilateral CAS $\geq 50 \%$ is associated with more favorable 
collateral status in acute LVO stroke patients. However, this does not translate into a better functional outcome at 90 days, which might be attributed to a higher rate of adverse events after MT. Although occurring rarely, CAS patients with unfavorable collaterals on pretreatment angiography face a particularly high risk of poor outcome three months after the intervention $(\approx 90 \%)$.

Preinterventional leptomeningeal collaterals have a significant impact on patients' clinical prognosis after MT. In line with earlier investigations, this study also shows that favorable collateral status on pretreatment angiography was associated with smaller postinterventional infarct size and a better functional outcome at 90 days after the intervention [5-7]. Conditions that could predict favorable leptomeningeal collaterals after acute cerebral artery occlusion are therefore of interest. In this context, chronic cerebral hypoperfusion was associated with improved cerebral collateral flow in experimental rat models and in patients with stenoocclusive disease of the carotid vasculature; and the effect increased with the degree of stenosis $[4,15,16]$. Moreover, repeated arterio-arterial (micro)embolism proceeding from aggressive carotid plaques could lead to recurrent and clinically silent cerebral ischemia, which might trigger better collaterals due to ischemic preconditioning [17].

Although recent studies only addressed primary collateral pathways in the circle of Willis, it seems plausible that CAS patients with acute intracranial LVO stroke would also be associated with favorable leptomeningeal collateral recruitment, which could further impact patients' clinical prognosis.

While two small studies presented divergent results on that topic, $[6,7]$ a very recent MR-CLEAN Registry subanalysis showed that CAS-related LVO stroke patients had a better collateral status than those with cardioembolic stroke, [8] which is in line with our work. In contrast to our findings, the latter study showed slightly better median 90-days $\mathrm{mRS}$ scores in their CAS subgroup. This might be attributed to methodological differences between both studies: From a pathophysiological perspective, we decided to include all patients without an indication of symptomatic carotid stenosis $\geq 50 \%$ (according to the NASCET criteria) in our non-CAS group. As we did not observe patients with ulcerated carotid plaques, most non-CAS patients should have had a proximal (cardio)embolic stroke etiology, which is frequently missed on routine stroke work-up (e.g. in case of paroxysmal atrial fibrillation). However, such initially cryptogenic stroke patients are younger and have less comorbidities compared to the standard cardioembolic stroke patients [18]. The exclusion of such patients as it was done by the MR-CLEAN investigators might therefore explain the reported baseline imbalances in their subgroup analysis [8]. In contrast, our study provides comparable subgroups (CAS versus non-CAS) regarding age, medical history and prestroke mRS. Of note, the effect on outcome presented in the MR-CLEAN Registry subanalysis remained relatively low as there was no statistically significant benefit for CAS patients in terms of 90-day post-stroke dependency or mortality rates [8]. The authors concluded that larger thrombi and difficulties in gaining intracranial access due to proximal stenosis might have caused longer thrombectomy procedures compromising the positive impact of good collaterals on clinical prognosis. Our study also shows a trend towards longer interventions in CAS-related strokes, but additionally draws attention to postinterventional adverse events (predominantly vessel re-occlusions and symptomatic ICH) in CAS-related thrombectomy patients.

The high percentage of vessel re-occlusion $(11 \%)$ we detected in our CAS subgroup might be also a result of more complex endovascular procedures (balloon dilatation: $43 \%$, stenting of CAS: $24 \%$ ) and residual stenosis leading to recurrent arterio-arterial embolism. Although data on vessel reocclusion in the early phase after MT are scarce, this finding is in line with a small retrospective study, which has shown rather high rates of postinterventional re-stenosis/occlusion and a poor prognosis in LVO stroke patients with tandem pathologies [19].

Symptomatic intracerebral bleeding was the second most common adverse event in our study and might be partly attributed to extracranial artery stenting in the CAS subgroup, which required more intense antiplatelet therapy (i.e. dual antiplatelets) after the intervention. In addition, carotid artery stenting might enlarge reperfusion injury, which seems generally more pronounced in chronically ischemic tissues due to disturbances of cerebral autoregulation [9].

Of note, this is the first study that casts light on acute LVO patients with unfavorable collaterals despite underlying CAS, as they are at very high risk for postinterventional adverse events (37\%) and functional dependency at 90 days (91\%). Although the number of patients in this subgroup was rather small, this finding should be considered in the clinical management of such patients.

The major limitation of this study is the retrospective design and the fact that no blinding regarding stroke etiology and collateral status was possible. However, during rating, neuroradiologists were blinded to clinical information including adverse events and outcome. We decided to abstain from volumetry, but instead used pre- and postinterventional CT or MRI based ASPECT Scores to estimate the acute and final infarct, which is more feasible in daily clinical routine.

Moreover, we did not analyze the Circle of Willis for anatomical variants, which could have affected our results and should be addressed in future studies on cerebral collaterals.

Another restriction was, that not all patients underwent multi-phase CT/MR-angiography $(\approx 34 \%)$. If collateral filling in the post venous phase occurs, collaterals could be 
underestimated when using the Tan Score in single-phase angiography. However, as this is not a frequent finding and collaterals were comparable to those of earlier investigations using multi-phase angiography, it should not have influenced our results to a major extent [20].

Finally, we cannot totally exclude that in few cases emboli broke off from the carotid stenosis leaving it less than $50 \%$. However, patients with minor carotid artery stenosis $<50 \%$ did not meet the established ultrasound criteria for aggressive plaques (i.e. ulceration, intraplaque hemorrhage, etc.), which should exclude a major effect on the results of this investigation.

Acknowledgements Open access funding provided by Medical University of Graz.

Funding None.

Availability of data and material Data and material from this study are available from the corresponding author upon reasonable request.

\section{Compliance with ethical standards}

Conflicts of interest The authors declare that they have no conflict of interest.

Ethical approval The study was approved by the ethics committee of the Medical University of Graz in accordance with the ethical standards laid down in the 1964 Declaration of Helsinki and its later amendments. Informed consent was waived by the ethics committee due to the retrospective nature of this study.

Informed consent All authors have read and approved the submitted manuscript, which has not been submitted elsewhere nor published elsewhere in whole or in part. No author reported a relevant conflict of interest relating to this paper.

Open Access This article is licensed under a Creative Commons Attribution 4.0 International License, which permits use, sharing, adaptation, distribution and reproduction in any medium or format, as long as you give appropriate credit to the original author(s) and the source, provide a link to the Creative Commons licence, and indicate if changes were made. The images or other third party material in this article are included in the article's Creative Commons licence, unless indicated otherwise in a credit line to the material. If material is not included in the article's Creative Commons licence and your intended use is not permitted by statutory regulation or exceeds the permitted use, you will need to obtain permission directly from the copyright holder. To view a copy of this licence, visit http://creativecommons.org/licenses/by/4.0/.

\section{References}

1. Goyal M, Menon BK, van Zwam WH, HERMES collaborators et al (2016) Endovascular thrombectomy after large-vessel ischaemic stroke: a meta-analysis of individual patient data from five randomised trials. Lancet 387:1723-1731

2. Bang OY, Saver JL, Kim SJ et al (2011) Collateral flow predicts response to endovascular therapy for acute ischemic stroke. Stroke 42:693-699
3. Bang OY, Saver JL, Kim SJ, UCLA-Samsung Stroke Collaborators et al (2011) Collateral flow averts hemorrhagic transformation after endovascular therapy for acute ischemic stroke. Stroke 42:2235-2239

4. Henderson RD, Eliasziw M, Fox AJ, Rothwell PM, Barnett HJ (2000) Angiographically defined collateral circulation and risk of stroke in patients with severe carotid artery stenosis. North American symptomatic carotid endarterectomy trial (nascet) group. Stroke 31:128-132

5. Sallustio F, Motta C, Pizzuto S et al (2017) CT angiography-based collateral flow and time to reperfusion are strong predictors of outcome in endovascular treatment of patients with stroke. J Neurointerv Surg 9:940-943

6. Elijovich L, Goyal N, Mainali S et al (2015) CTA collateral score predicts infarct volume and clinical outcome after endovascular therapy for acute ischemic stroke: a retrospective chart review. J Neurointerv Surg 8:559-562

7. Rebello LC, Bouslama M, Haussen DC et al (2017) Stroke etiology and collaterals: atheroembolic strokes have greater collateral recruitment than cardioembolic strokes. Eur J Neurol 24:762-767

8. Guglielmi V, LeCouffe NE, Zinkstok SM, MR-CLEAN-RegistryInvestigators-Group Authors et al (2019) Collateral circulation and outcome in atherosclerotic versus cardioembolic cerebral large vessel occlusion. Stroke 29:119026299

9. Kneihsl M, Niederkorn K, Deutschmann H et al (2018) abnormal blood flow on transcranial duplex sonography predicts poor outcome after stroke thrombectomy. Stroke 49:2780-2782

10. Aboyans V, Ricco JB, Bartelink MEL, ESC Scientific Document Group et al (2018) 2017 ESC Guidelines on the Diagnosis and Treatment of Peripheral Arterial Diseases, in collaboration with the European Society for Vascular Surgery (ESVS): document covering atherosclerotic disease of extracranial carotid and vertebral, mesenteric, renal, upper and lower extremity arteriesEndorsed by: the European Stroke Organization (ESO)The Task Force for the Diagnosis and Treatment of Peripheral Arterial Diseases of the European Society of Cardiology (ESC) and of the European Society for Vascular Surgery (ESVS). Eur Heart J 39:763-816

11. Barnett HJM, Taylor DW, Haynes RB, North American Symptomatic Carotid Endarterectomy Trial Collaborators et al (1991) Beneficial effect of carotid endarterectomy in symptomatic patients with high-grade carotid stenosis. N Engl J Med 325:445-453

12. Tan IY, Demchuk AM, Hopyan J et al (2009) CT angiography clot burden score and collateral score: correlation with clinical and radiologic outcomes in acute middle cerebral artery infarct. AJNR Am J Neuroradiol 30:525-531

13. Von Kummer R, Broderick JP, Campbell BC et al (2015) The Heidelberg bleeding classification: classification of bleeding events after ischemic stroke and reperfusion therapy. Stroke 46:2981-2986

14. Yu S, Liebeskind DS, Dua S, UCLA Stroke Investigators et al (2015) Postischemic hyperperfusion on arterial spin labeled perfusion MRI is linked to hemorrhagic transformation in stroke. J Cereb Blood Flow Metab 35:630-637

15. Busch HJ, Buschmann IR, Mies G, Bode C, Hossmann KA (2003) Arteriogenesis in hypoperfused rat brain. J Cereb Blood Flow Metab 23:621-628

16. van Everdingen KJ, Visser GH, Klijn CJ, Kappelle LJ, van der Grond J (1998) Role of collateral flow on cerebral hemodynamics in patients with unilateral internal carotid artery occlusion. Ann Neurol 44:167-176

17. Khan MB, Hafez S, Hoda MN et al (2018) Chronic remote ischemic conditioning is cerebroprotective and induces vascular remodeling in a VCID model. Transl Stroke Res 9:51-63 
18. Zathar Z, Karunatilleke A, Fawzy AM, Lip GYH (2019) Atrial fibrillation in older people: concepts and controversies. Front Med 6:175

19. Soize S, Kadziolka K, Estrade L, Serre I, Barbe C, Pierot L (2014) Outcome after mechanical thrombectomy using a stent retriever under conscious sedation: comparison between tandem and single occlusion of the anterior circulation. J Neuroradiol 41:136-142
20. Volders D, Shewchuk JR, Marangoni M, Ni Mhurchu E, Heran M (2019) Beyond the collaterals: additional value of multiphase CTA in acute ischemic stroke evaluation. Neuroradiol J. https:// doi.org/10.1177/1971400919845361 